\title{
Instanton size distributions from calibrated cooling
}

\author{
C. Michael ${ }^{\mathrm{a}}$ and P.S. Spencer ${ }^{\mathrm{b}} *$ \\ aDAMTP, University of Liverpool, PO Box 147, Liverpool L69 3BX, UK \\ ${ }^{\mathrm{b}}$ Research Institute for Theoretical Physics (TFT), Siltavuorenpenger 20C, 00014 Helsinki, Finland.
}

Using an under-relaxed cooling algorithm we investigated the vacuum in the $2 d O(3)$ model and $4 d$ pure gauge $S U(2)$. We calibrated the amount of cooling performed to have similar physical effect at different lattice spacings. [Liverpool preprint: LTH 337; TFT preprint: HU-TFT-94-45, hep-lat/9411015]

\section{Introduction}

Topology is an important aspect of many lattice field theories, in particular QCD, where a non-zero value of the topological susceptibility is necessary to solve the $U(1)$ problem and explain the mass of the $\eta^{\prime}$ meson. For QCD the topology is a consequence of the existence of instanton solutions of the theory, so simpler theories than QCD that share the property of instanton solutions have long been of interest.

In this paper we present work carried out on two such simpler theories: $2 d O(3)$ and $4 d$ pure gauge $S U(2)$. In both of these theories we extracted information about the underlying longrange structure of the theory by cooling, a process which has been widely used for this purpose. It was immediately apparent to us that, as we wished to discuss the underlying physics, we would need to compare results obtained at different lattice spacings and we would therefore need to calibrate the cooling we used to have the same physical effect across the range of $a$ that we used; performing the same, arbitrary number of cooling sweeps at each value of $a$ would have different physical effects at different couplings, rendering a comparison of the results meaningless - different cooling levels would mean the gross structure of the configuration would be different, and it is on this structure that we wish to make calculations and compare results.

\footnotetext{
${ }^{*}$ Talk given by PSS. Work carried out as part of the EC Programme "Human Capital and Mobility" - project number ERB-CHRX-CT92-0051.
}

\section{The $O(3)$ Model}

The $2 d O(3)$ model shares many characteristics with QCD: it is asymptotically free, with dynamic mass generation and instanton solutions due in this case to the homotopy class of windings from $S_{2} \rightarrow S_{2}$.

Using a standard stereographic projection the single instanton solution is given by:

$\omega(z, \bar{z})=\frac{\phi_{1}+\mathrm{i} \phi_{2}}{1-\phi_{3}}=\frac{\rho}{z-r}, z=x+\mathrm{i} t$

with both $\rho$ and $r$ complex, and $\phi_{i}$ the $i$ th component of $\phi$. The action of this continuum field configuration is $S_{I}=4 \pi / g^{2}$. (It should be noted that on a torus there is no single-instanton solution [3.) In general multi-instanton solutions are given by analytic functions of $\omega$, ie $\partial_{\bar{z}} \omega=0$.

Unfortunately, the lattice version of the $O(3)$ model has problems, as the theory is dominated by short-range fluctuations [2], and formulating the theory on a lattice imposes a minimum size to these fluctuations, and so the important contribution from those smaller than $a$ is lost. Furthermore, additional unphysical contributions arise from lattice artefacts of size $\mathcal{O}(a)$. So on the one hand results obtained from cooling would not have the contribution from the physical ultraviolet fluctuations, but on the other hand they would not be corrupted by the unphysical artefacts, and measurements of objects of moderate size, $a \ll \rho \ll L$, should be reliable. On these grounds we decided to investigate the size distribution of instantons. 
Table 1

Details of the cooling used for $O(3)$. Data calculated on a $64^{2}$ lattice.

\begin{tabular}{ccccc}
\hline$g^{2}$ & $m a$ & $\xi / 3$ & $\xi / 2$ & $2 \xi / 3$ \\
\hline 0.80 & $0.261(1)$ & 28 & 79 & 220 \\
0.84 & $0.318(1)$ & 17 & 45 & 106 \\
1.00 & $0.551(1)$ & 5 & 13 & 24 \\
\hline
\end{tabular}

We used an under-relaxed cooling given by:

$\phi_{x}^{\prime}=\alpha \phi_{x}+\phi_{x}^{F}, \phi_{x}^{F}=\sum_{\mu}\left(\phi_{x+\mu}+\phi_{x-\mu}\right)$

with $\phi_{x}^{\prime}$ normalised to unit length. This was used for two reasons: firstly, as a deterministic update it is much faster to compute, and secondly: the parameter $\alpha$ allows us to control the severity of the cooling, though a trade-off must be made between the gentler cooling associated with larger values of $\alpha$ and the greater number of sweeps then needed to reach a given physical state. We eventually decided that $\alpha=2$ was a good compromise between too many sweeps and too harsh a cool. $\mathcal{O}(a)$ effects in the lattice $O(3)$ action mean that lattice instantons are unstable under cooling and will be shrunk and eventually annihilated by prolonged cooling. We decided to calibrate our cooling for the $O(3)$ model by measuring the number of cooling sweeps at different values of $\alpha$ required to annihilate a configuration generated from a discretised form of eq. 1. We looked at three levels of cooling at each of three couplings: $g^{2}=1.00,0.84,0.80$ corresponding to mass gaps of $m a=0.551(1), 0.381(1), 0.261(1)$, the levels being those required to remove objects of size one-third, one half and two-thirds the correlation length $\xi=1 / m$ at each value of $g^{2}$. The number of sweeps needed are given in table 1. We found that $S / S_{I} V$ calculated on the cooled configurations was consistent across $g^{2}$ for each of the cooling levels we used, and took this as evidence for correct calibration of our cooling.

\section{Pure gauge $S U(2)$}

For $S U(2)$ we again used an under-relaxed cooling, this time given by:

$U_{x, \mu}^{\prime}=\alpha U_{x, \mu}+\Sigma_{x, \mu}$

with $U_{x, \mu}^{\prime}$ normalised to lie in $S U(2)$ and $\Sigma_{x, \mu}$ the sum of the 'staples' around $U_{x, \mu}$.

When we came to extend the techniques we developed for $O(3)$ to the case of $4 d S U(2)$ we ran into the problem that the lattice $S U(2)$ instantons are much more stable under cooling than their $O(3)$ counterparts - so stable in fact that had we naïvely taken the same criterion as earlier, we would have cooled the configurations beyond any region where we would wish to study the vacuum, and in certain cases removed all the physics present in the uncooled configuration. In light of this we changed our approach: as we wish to consider the vacuum as quantum fluctuations around classical solutions, it makes sense, taking into account the stability of the lattice instantons, to calibrate the cooling by first constructing classical solutions, introducing quantum fluctuations by a few Monte Carlo updates and then cooling until the original configuration is recovered. This procedure is performed a number of times and the average number of sweeps needed to recover the initial state is taken as the calibration. Preliminary studies of this on $16^{4}$ lattices at $\beta=2.4$ and $24^{4}$ at $\beta=2.5$ have proved promising, and the data imply that 61 sweeps at $\alpha=2$ is sufficient to remove the quantum fluctuations without overly disturbing the underlying structure on a $16^{4}$ lattice at $\beta=2.4$.

\section{Instanton Size Distributions}

In order to calculate the sizes of instantons we looked for connected regions around local maxima in the action density for which the action density was not less than half the value at the maximum under consideration; 2-dimensional regions in $O(3)$ and 4-dimensional for $S U(2)$. We then took the appropriate root of this volume to obtain a size parameter. Predictions of the instanton size distribution in both $O(3)$ and $S U(2)$ can be derived from the dilute instanton gas model 
(see, for example, ref. [1]). For $O(3)$ the model predicts

$\frac{1}{S_{I} V} \frac{d S}{d \rho} \sim \frac{1}{\rho}$

whereas our data, presented in [3] and summarised in figure 1. indicate a much stronger UV dependence and a distribution $\sim \rho^{-3}$. For $S U(2)$, the dilute gas predicts an infra-red divergence and

$$
\frac{1}{S_{I} V} \frac{d S}{d \rho} \sim \rho^{7 / 3}
$$

It is unclear whether our preliminary data, shown in figure 1 are in agreement with this. Certainly they indicate no ultra-violet divergence, and it appears that the very smallest instantons are all but absent.

For $O(3)$, while the finite lattice spacing reduces any signal below $\rho \sim 1.5 \xi$, we see a rapid decrease at larger $\rho$. For $S U(2)$ this decrease is less rapid. The physical volumes here are approximately equal, with $L \sim 16 \xi$, so we take this difference in large $\rho$ behaviour to be a physical effect.

For $O(3)$ we also looked at the separation of pairs of instantons and anti-instantons, and found evidence for an interaction: the closest separation of unlike pairs of objects was significantly smaller than that of like pairs. At each value of $g^{2}$ we found that unlike pairs had a closest separation only $70 \%$ that of like pairs. This interaction, and the form of the distribution we found show no support for the dilute gas model for $O(3)$ instantons on the lattice. The corresponding $S U(2)$ measurements are in progress.

\section{Conclusions}

We have given an argument in favour of calibrating cooling to have a given physical effect, and altering the number of sweeps as the lattice spacing is altered. We have also presented data calculated using calibrated cooling and shown how it is consistent across a range of couplings.

\section{REFERENCES}

1. Solitons and Instantons, R. Rajaraman, North Holland (1982).

2. M. Lüscher, Nuc. Phys. B200, 61 (1982).
3. C. Michael, P.S. Spencer, Liverpool preprint LTH-331, to appear in Phys. Rev. D. 

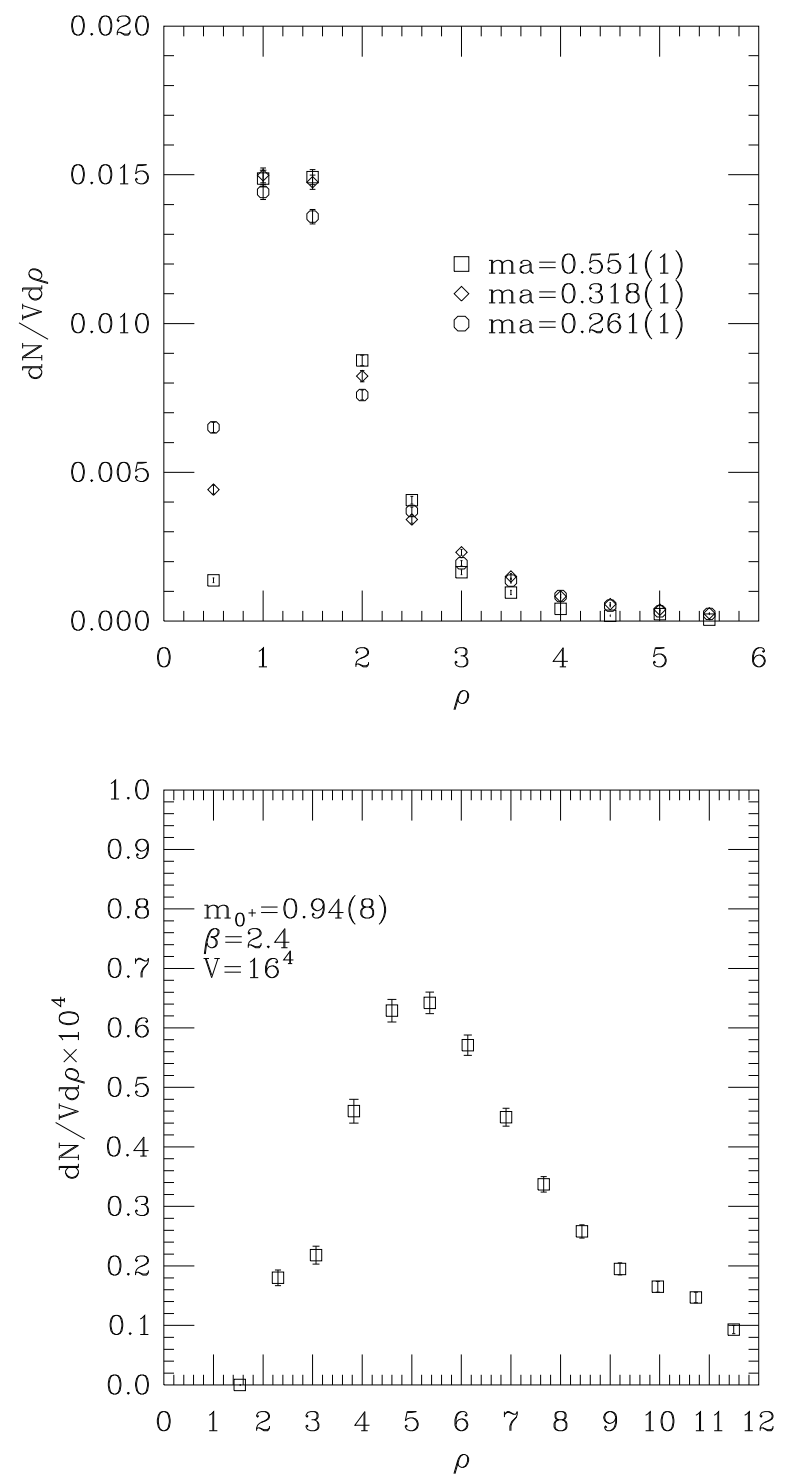

Figure 1. The distributions of instanton size calculated for $O(3)$ from 1000 configurations for each value of $a$ on a $64^{2}$ lattice (upper plot), and for $S U(2)$ from 1000 configurations at $\beta=2.4$ on a $16^{4}$ lattice (lower plot). Instanton sizes are given in units of the correlation length, $\xi=1 / m$; for $S U(2), m=m_{0^{+}}$. We define $N=S / S_{I}$ 